Article

\title{
Prognostic Significance of ROR2 Expression in Patients with Urothelial Carcinoma
}

\author{
Cheng-Fa Yeh ${ }^{1,2}$, Ti-Chun Chan ${ }^{3,4}$, Hung-Lung Ke ${ }^{5,6,7}$, Tzu-Ju Chen ${ }^{8}$, Li-Ching Wu ${ }^{8}$, Hsiang-Ying Lee ${ }^{5,6,7}$, \\ Yu-Ching Wei ${ }^{9,10}$, Wen-Jeng $\mathrm{Wu}^{5,6,11}$, Chien-Feng $\mathrm{Li}^{3,4}$ and Wei-Ming $\mathrm{Li}^{5,6,11,12, *}$
}

1 Division of General Internal Medicine, Chi Mei Medical Center, Tainan 710, Taiwan; u802091@gmail.com

2 Department of Environment Engineering and Science, Chia Nan University of Pharmacy and Science, Tainan 717, Taiwan

3 Department of Medical Research, Chi Mei Medical Center, Tainan 710, Taiwan; chloe0304@nhri.edu.tw (T.-C.C.); cfli@mail.chimei.org.tw (C.-F.L.)

4 National Institute of Cancer Research, National Health Research Institutes, Tainan 704, Taiwan

5 Department of Urology, Kaohsiung Medical University Hospital, Kaohsiung 807, Taiwan; hlke@kmu.edu.tw (H.-L.K.); hsiangying@kmu.edu.tw (H.-Y.L.); wejewu@kmu.edu.tw (W.-J.W.)

6 Department of Urology, School of Medicine, College of Medicine, Kaohsiung Medical University, Kaohsiung 807, Taiwan

7 Department of Urology, Kaohsiung Municipal Ta-Tung Hospital, Kaohsiung 801, Taiwan

8 Department of Clinical Pathology, Chi Mei Medical Center, Tainan 710, Taiwan; a108n2@mail.chimei.org.tw (T.-J.C.); 540012@mail.chimei.org.tw (L.-C.W.)

9 Department of Pathology, School of Medicine, College of Medicine, Kaohsiung Medical University, Kaohsiung 807, Taiwan; ycwei@kmu.edu.tw

10 Department of Pathology, Kaohsiung Municipal Ta-Tung Hospital, Kaohsiung 801, Taiwan

11 Center for Liquid Biopsy and Cohort Research, Kaohsiung Medical University, Kaohsiung 807, Taiwan

check for

updates

Citation: Yeh, C.-F.; Chan, T.-C.; Ke, H.-L.; Chen, T.-J.; Wu, L.-C.; Lee, H.-Y.; Wei, Y.-C.; Wu, W.-J.; Li, C.-F.; Li, W.-M. Prognostic Significance of ROR2 Expression in Patients with Urothelial Carcinoma. Biomedicines 2021, 9, 1054. https:// doi.org/10.3390/biomedicines9081054

Academic Editor: Massimo Moro

Received: 18 July 2021

Accepted: 17 August 2021

Published: 20 August 2021

Publisher's Note: MDPI stays neutral with regard to jurisdictional claims in published maps and institutional affiliations.

Copyright: (c) 2021 by the authors. Licensee MDPI, Basel, Switzerland. This article is an open access article distributed under the terms and conditions of the Creative Commons Attribution (CC BY) license (https:/ / creativecommons.org/licenses/by/ $4.0 /)$.
12 Department of Urology, Ministry of Health and Welfare Pingtung Hospital, Pingtung 900, Taiwan

* Correspondence: wmli@kmu.edu.tw; Tel.: +886-7-312-1101 (ext. 6694)

\begin{abstract}
We investigated the association of receptor tyrosine kinase-like orphan receptor 2 (ROR2) expression with clinicopathological features and oncologic outcomes in large urothelial carcinoma (UC) of the upper tract (UTUC) and urinary bladder (UBUC) cohorts. Through transcriptomic profiling of a published dataset (GSE31684), ROR2 was discovered to be the most upregulated gene during UC progression, focusing on the JNK cascade (GO:0007254). Initially, the evaluation of ROR2 mRNA expression in 50 frozen UBUCs showed significantly upregulated levels in highstage UC. Moreover, high ROR2 immunoexpression significantly correlated with high tumor stage, high tumor grade, lymph node metastasis, and vascular invasion (all $p<0.05$ ). In multivariate analysis, after adjusting for standard clinicopathological features, ROR2 expression status was an independent prognosticator of cancer-specific survival and metastasis-free survival in UTUC and UBUC (all $p<0.01$ ). In the subgroup analysis, it also significantly predicted bladder tumor recurrence in non-muscle invasive UBUC. Furthermore, the GO enrichment analysis showed that fatty acid, monocarboxylic acid, carboxylic acid metabolic processes, negative regulation of neutrophil migration, and negative regulation of granulocyte and neutrophil chemotaxis were significantly enriched by ROR2 dysregulation. In conclusion, high ROR2 immunoexpression was associated with aggressive pathological characteristics in UC and independently predicted worse prognosis, suggesting it could play roles in clinical risk stratification and therapy decisions.
\end{abstract}

Keywords: ROR2; bladder cancer; upper tract urothelial carcinoma; survival; prognosis

\section{Introduction}

Urothelial carcinoma (UC) of the upper tract (ureter and renal pelvis) (UT) and urinary bladder (UB) is one of the most frequently occurring malignancies worldwide. UBUC is the 10th most common malignancy globally, with 573,278 estimated new cases in both sexes in $2020,37 \%$ of which $(212,536)$ are predicted to be lethal [1]. Tobacco smoking and 
occupational exposure to aromatic amines or polycyclic are the main environmental risk factors for UC [2-4]. Recent meta-analyses have demonstrated that current and former smokers are at an increased risk of UC. Dose-response analyses indicate that even after long-term smoking cessation, an elevated risk of UC remains [5]. Although family history and heritable gene variants seem to have little impact, genetic studies have demonstrated a potential role of genetic predisposition to urothelial carcinogenesis [6,7].

For UBUC, most non-muscle-invasive bladder cancer (NMIBC) can be treated by the transurethral resection of the bladder tumor (TURBT) and subsequent intravesical instillations [2,3]. Aggressive management, including radical cystectomy, bladder preservation treatment, and perioperative chemotherapy or immunotherapy, are suggested for muscle-invasive bladder cancer (MIBC) or high-risk NMIBC [2,3]. For UTUC, radical nephroureterectomy (RNU) is the standard management of high-risk cancers; kidneysparing surgery should be considered in all low-risk cases or in select patients with serious renal insufficiency or solitary kidney [4]. Although surgical techniques and therapeutic modalities have significant advancements, the prognosis of patients with UC has not significantlyimproved in the lastthree decades [2-4]. Therefore, it is vital to discover new prognostic biomarkers and elucidate the UC progression mechanism.

Emerging evidence shows that c-Jun N-terminal kinase (JNK) promotes tumor progression and is involved in various cancers [8,9]. JNK-associated signaling pathways also modulate metabolic reprogramming, cancer stem cells, tumor proliferation, and migration [8-11]. Focusing on the genes belonging to the JNK cascade (GO:0007254) and associated cancer progression, data mining was performed on a public dataset of bladder cancer. These genes are listed in Tables S1-S5. Some of them, such as MLK3, TAK1, ASK1, $M K K 4, M K K 7$, JNK1, and JNK2, have been demonstrated to regulate cell proliferation, cell survival, cell differentiation, and cell renewal $[8,9]$.We found that receptor tyrosine kinase-like orphan receptor 2 (ROR2) was the most upregulated gene during bladder cancer progression, from NMIBC to MIBC.

ROR2, a novel Wnt receptor, is normally expressed at high levels during development and morphogenesis and plays a key role in chondrogenesis, plate development, chondrogenesis, and growth plate development [12]. It is a highly pleiotropic receptor with a complex role in human carcinogenesis. The upregulation of ROR2 has been established in a multitude of tumor types, such as osteosarcoma, prostate cancer, and renal cell carcinoma [13-15]. In contrast, ROR2 acts to suppress carcinogenesis in colon cancer and hepatocellular carcinoma $[16,17]$. However, the roles of ROR2 in UC have not been well studied. We aimed to elucidate the association of ROR2 with clinicopathological features and the prognostic impact of UC in our cohort.

\section{Materials and Methods}

\subsection{Public Data}

The UBUC patient data (GSE31684) retrieved from the Gene Expression Omnibus (GEO) data repository was a transcriptomic public microarray dataset measured on the Affymetrix Human Genome HG-U133 Plus 2.0 array. We processed the data as previously reported [18,19]. Raw files were imported into the Nexus Expression 3 software (BioDiscovery, El Segundo, CA, USA) without pre-selection to computerize the expression level. To identify the cancer progression-associated genes, we calculated the differentially expressed genes (DEGs) focusing on the JNK cascade (GO:0007254) between MIBC and NMIBC. We selected the top six DEGs $(p<0.01$ and $\mid \log 2$ ratio $\mid>0.5)$ for further research.

\subsection{Patient Data and Tissues}

After Institutional Review Board approval (IRB10501-005) on 4 February 2016, we identified 635 UC patients, and 295 UBUC and 340 UTUC patients were treated with surgery with curative intent between 1998 and 2004. None received neoadjuvant chemotherapy or radiotherapy before surgery. The performance and extent of lymphadenectomy were based on the surgeon's discretion. All surgical specimens were processed per the standard 
pathological procedures. The pathological stage was reassigned on the basis of the 2010 AJCC/UICC classification, and tumor grade was evaluated using the 2004WHO/ISUP consensus criteria. Comprehensive clinical, pathological, and follow-up data elements were retrospectively reviewed for each patient.

\subsection{Quantitative Real-Time PCR}

Using the Total RNA Purification Kit (GeneMark, Atlanta, GA, USA), total RNA extraction was performed, and cDNA was obtained using the Maxima First Strand cDNA Synthesis Kit (Thermo Fisher Scientific, Waltham, MA, USA) according to the manufacturer's instructions. Quantitative real-time PCR (qRT-PCR) was performed on a StepOne Plus System (Applied Biosystems, Waltham, MA, USA) as previously described [18,19]. We calculated the fold expression of ROR2 relative to the adjacent non-tumor urothelium using the $\triangle \triangle \mathrm{CT}$ method after normalization to the endogenous control gene, POLR2A.The following TaqMan ${ }^{\mathrm{TM}}$ Gene Expression assay probes (Thermo Fisher Scientific, Waltham, MA, USA) were used: ROR2 (assay ID:Hs00896176_m1, Catalog No.:4331182) and POLR2A (assay ID:Hs01108291_m1, Catalog No.:4331182).

\subsection{Immunohistochemistry}

We followed the standard immunohistochemistry (IHC) protocols as described previously [20,21]. Four-micrometer-thin sections were cut from formalin-fixed, paraffinembedded tissue blocks. The samples were then incubated with anti-ROR2 primary antibody (1:100, ab-218105, Abcam). We detected IHC reactions using the DakoREALEnVision ${ }^{\mathrm{TM}}$ detection system. Slides were analyzed by a manual reading of two dedicated pathologists who were blinded to the clinicopathological features and patient outcomes. They appraised the intensity (0:negative; $1+$ :weak; $2+$ :moderate; $3+$ : strong) and percentage ( 0 to $100 \%$ ) of positive immunostaining UC cells to generate the H-score, using the following equation: H-score $=1 \times(\%$ of weakly stained cells $)+2 \times(\%$ of moderately stained cells $)+3 \times(\%$ of strongly stained cells). An H-score between 0 and 300 was obtained, wherein 300 equals $100 \%$ of the tumor cell staining corresponding to strongly positive $(3+)$ staining. We divided immunoreactivity into low and high expression levels using the median H-score.

\subsection{Gene Ontology (GO) Enrichment Analysis}

We used the gene expression information from the TCGA_BLCA database of the cBioPortal platform (https://www.cbioportal.org/) accessed on 1 December 2020 and screened for ROR2 positive and negative co-expression genes. Spearman correlation coefficient was calculated. The top 500 co-expressed genes were selected for GO enrichment analysis to understand the function of ROR2 co-expression genes on the gene-ontology website (https: / / geneontology.org/) accessed on 1 December 2020, which was powered by the PANTHER classification system.

\subsection{Statistical Analysis}

Associations between ROR2 expression and patient or UC characteristics were determined using a chi-square test. Oncologic outcomes, including disease-specific survival (DSS), metastasis-free survival (MFS), and bladder recurrence-free survival (BRFS), were measured from the date of surgery to the date of events that occurred. Survivors at the end of the study were censored at the date of the last follow-up. These outcomes were compared using Kaplan-Meier methods stratified by the ROR2 expression status (high vs. low) using the log-rank statistic. We used multivariate Cox proportional hazard regression models to identify the independent predictors of oncologic outcomes (DSS, MFS, and BRFS). All statistical analyses were conducted using SPSS (version 17.0; IBM, Armonk, NY, USA). All tests were 2 -sided, and $p$ values $<0.05$ were considered statistically significant. 


\section{Results}

\subsection{Data Mining of Significantly Altered Genes Belong to the JNK Cascade in UC Progression}

Through transcriptomic profiling of a GEO dataset (GSE31684), we identified the top six significantly dysregulated genes belonging to the JNK cascade associated with muscle invasion in UBUC (Table 1 and Figure 1A). The prognostic potentials of these genes were assessed using GEPIA databases. Only high ROR2 expression is related to worse overall survival $(p=0.022)$ and disease-free survival $(p=0.024)$ (Figure 1B). The expression status of the other five genes is not associated with patient outcomes (Figure S1). Therefore, we selected ROR2 for further study. Initially, the ROR2 transcript was evaluated in 50 snap-frozen UBUC tissues. ROR2 mRNA showed significantly high expression in MIBC $(p<0.001)$, signifying its function in UC aggressiveness (Figure 2A). These findings prompted us to further study the correlations between the ROR2 protein level and clinicopathological features and its prognostic roles in our large, well-characterized UTUC and UBUC cohorts.

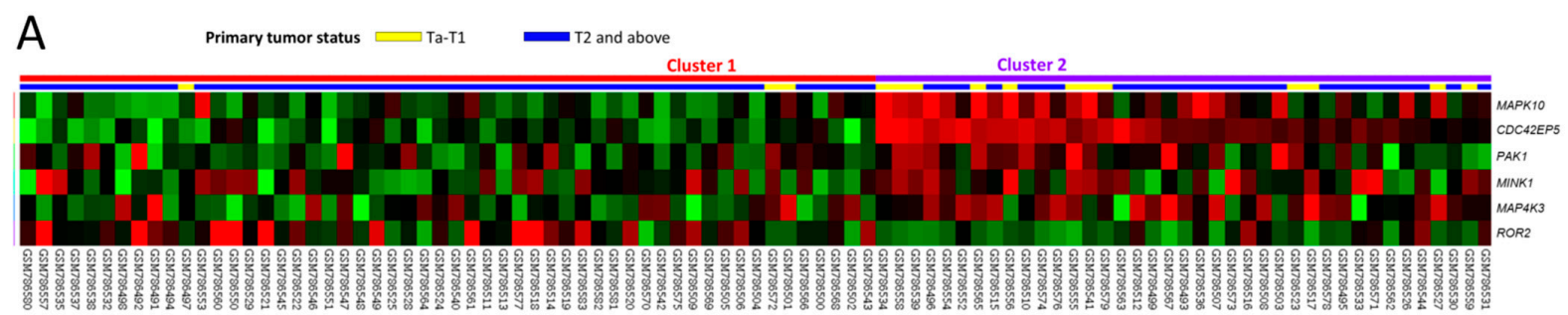

B

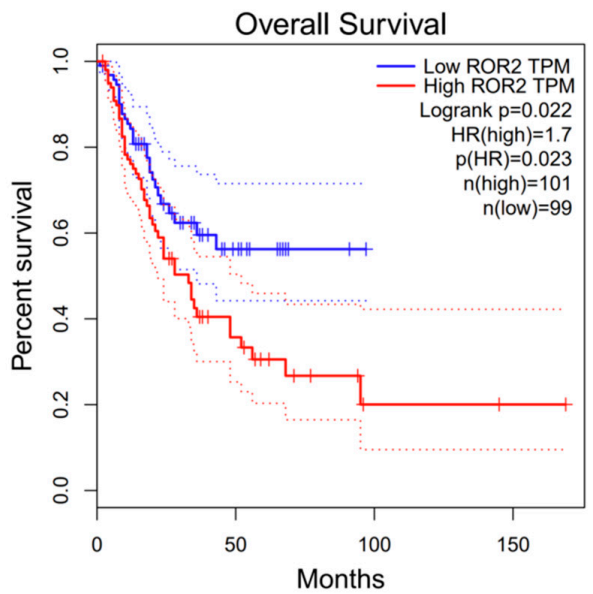

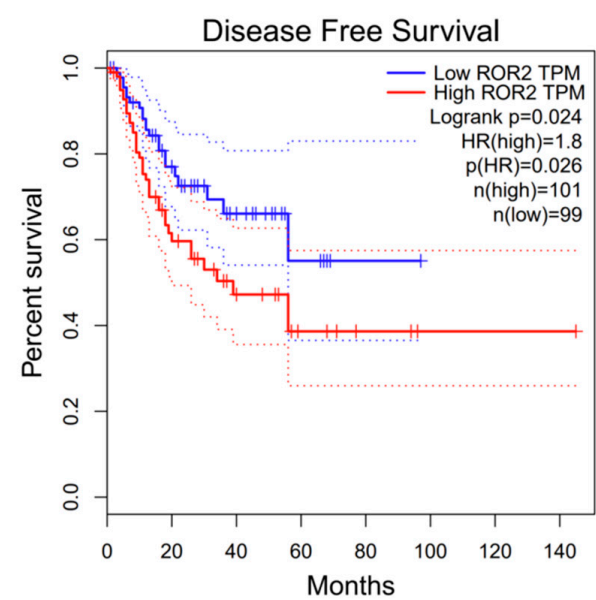

Figure 1. Transcriptomic profiling and survival analysis. (A) Expression profiles of genes associated with the progression of UBUC (T2-4 vs. Ta-1) focusing on JNK cascade from a published transcriptome (GSE31684) in Gene Expression Omnibus. ROR2 is the most significantly upregulated gene. (B) Using GEPIA databases, high ROR2 expression was remarkably related to worse overall survival $(p=0.022)$ and disease-free survival $(p=0.024)$ in UBUC.

\subsection{Descriptive Characteristics and Association with ROR2 Status}

Table 2 shows the association between ROR2 expression and clinicopathological features. Representative examples of ROR2 staining are shown in Figure 2B.

In the UTUC group, we included 340 patients, with $53.5 \%$ of male patients, and the mean age at the time of surgery was $65.58 \pm 9.89$ years. Gender distribution, age, tumor location, multifocality, perineural invasion, and mitotic rate were similar between the high and low expression groups. High ROR2 expression was more often associated with advanced pathologic T stage $(p<0.001)$, nodal metastasis $(p<0.001)$, high histological grade $(p=0.008)$ and vascular invasion $(p<0.001)$.

We included 295 patients in the UBUC cohort. The median age was 67 years, and most patients were men (73.2\%). There was no difference in sex, age, lymph node status, perineural invasion, and mitotic rate between patients with high and low ROR2expression. 
Tumors with high ROR2 expression by IHC showed a high primary tumor stage $(p<0.001)$, high histological grade $(p<0.001)$, and vascular invasion $(p=0.045)$.

A

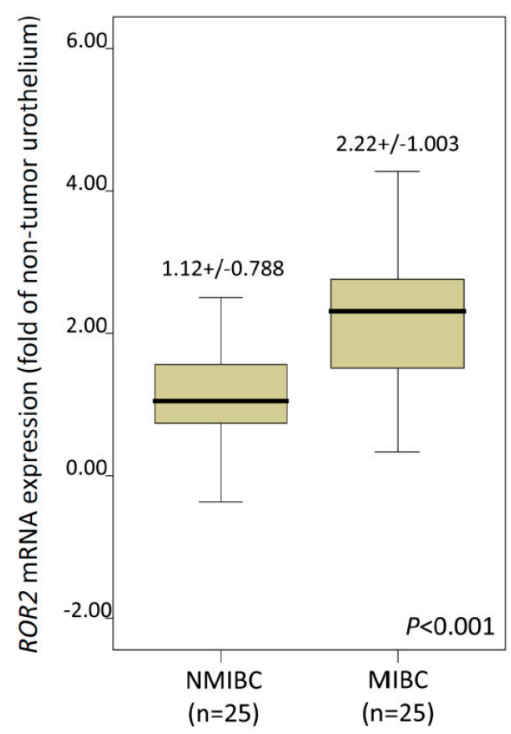

B Non-invasive UC

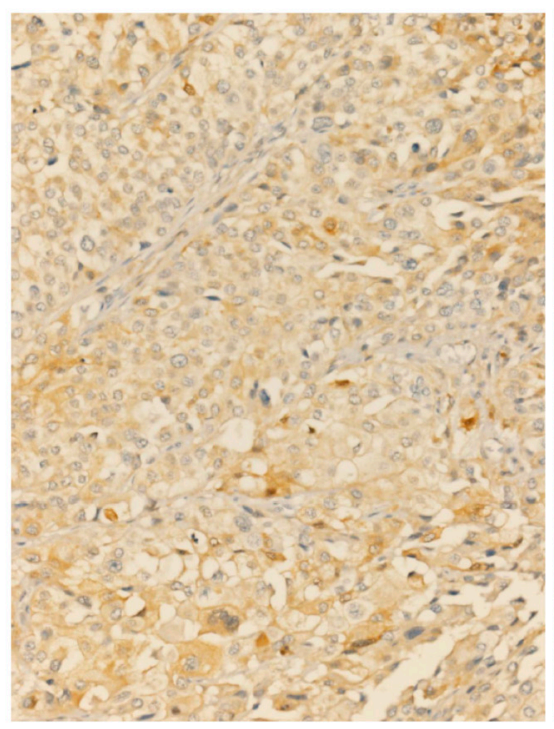

Invasive UC

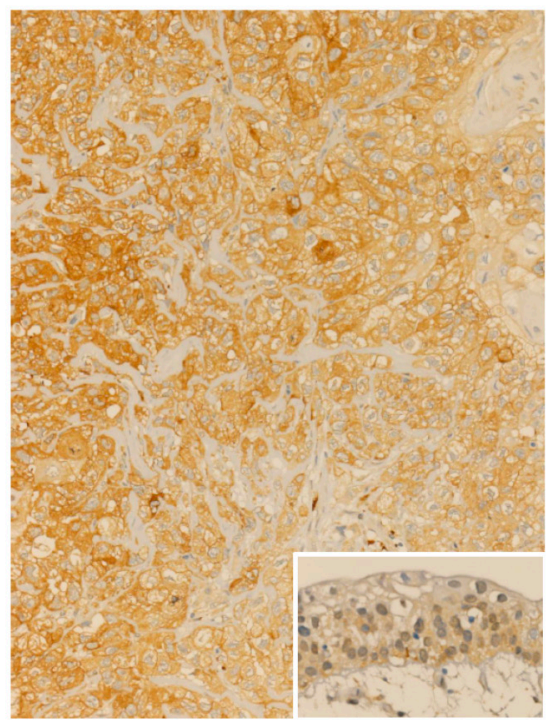

Figure 2. Expression of ROR2 mRNA and protein in urothelial carcinoma specimens. (A) ROR2 mRNA level was significantly increased in MIBC (pT2-T4) using qRT-PCR. (B) Invasive UC showed high ROR2 expression using immunohistochemistry (normal urothelium in the inset) (magnification $\times 2000$ ).

Table 1. The top six significantly dysregulated genes belonging to the JNK cascade are associated with muscle invasion in UBUC (GSE31684).

\begin{tabular}{|c|c|c|c|c|c|}
\hline \multirow{2}{*}{ Probe } & \multicolumn{2}{|c|}{ Comparing MIBC vs. NMIBC } & \multirow{2}{*}{ Gene Symbol } & \multirow{2}{*}{ Gene Title } & \multirow{2}{*}{ Biological Process } \\
\hline & Log Ratio & $p$-Value & & & \\
\hline 204813_at & -0.9123 & 0.0024 & MAPK10 & $\begin{array}{l}\text { mitogen-activated } \\
\text { protein kinase } 10\end{array}$ & $\begin{array}{l}\text { JNK cascade, protein amino acid } \\
\text { phosphorylation, signal transduction }\end{array}$ \\
\hline 205578_at & 0.6393 & $<0.0001$ & ROR2 & $\begin{array}{l}\text { receptor tyrosine } \\
\text { kinase-like orphan } \\
\text { receptor } 2\end{array}$ & $\begin{array}{l}\text { JNK cascade, Wnt receptor signaling } \\
\text { pathway; calcium modulating pathway, } \\
\text { cartilage condensation, cell differentiation, } \\
\text { embryonic genitalia morphogenesis, } \\
\text { multicellular organismal development, } \\
\text { protein amino acid phosphorylation, signal } \\
\text { transduction, skeletal development, } \\
\text { somitogenesis }\end{array}$ \\
\hline 214246_x_at & -0.5616 & 0.0083 & MINK1 & $\begin{array}{l}\text { misshapen-like } \\
\text { kinase } 1 \text { (zebrafish) }\end{array}$ & $\begin{array}{l}\text { JNK cascade, multicellular organismal } \\
\text { development, negative thymic T cell } \\
\text { selection, protein amino acid } \\
\text { phosphorylation, protein kinase cascade, } \\
\text { response to stress }\end{array}$ \\
\hline 218311_at & -0.5919 & 0.0072 & MAP4K3 & $\begin{array}{l}\text { mitogen-activated } \\
\text { protein kinase kinase } \\
\text { kinase kinase } 3\end{array}$ & $\begin{array}{l}\text { JNK cascade, protein amino acid } \\
\text { phosphorylation, protein kinase cascade, } \\
\text { response to stress }\end{array}$ \\
\hline 227850_x_at & -1.6026 & $<0.0001$ & CDC42EP5 & $\begin{array}{c}\text { CDC42 effector } \\
\text { protein (Rho GTPase } \\
\text { binding) } 5\end{array}$ & $\begin{array}{l}\text { JNK cascade, Rho protein signal } \\
\text { transduction, positive regulation of actin } \\
\text { filament polymerization, positive } \\
\text { regulation of pseudopodium formation, } \\
\text { regulation of cell shape }\end{array}$ \\
\hline 230100_x_at & -0.6927 & 0.0011 & PAK1 & $\begin{array}{c}\mathrm{p} 21 / \mathrm{Cdc} 42 / \text { Rac1- } \\
\text { activated kinase } 1 \\
\text { (STE20 homolog; } \\
\text { yeast) }\end{array}$ & $\begin{array}{l}\text { ER-nuclear signaling pathway, JNK } \\
\text { cascade, apoptosis, cytoskeleton } \\
\text { organization and biogenesis, dendrite } \\
\text { development, protein amino acid } \\
\text { phosphorylation }\end{array}$ \\
\hline
\end{tabular}


Table 2. Correlations between ROR2 expression and other important clinicopathological parameters in urothelial carcinomas.

\begin{tabular}{|c|c|c|c|c|c|c|c|c|c|}
\hline \multirow{3}{*}{ Parameter } & \multirow{3}{*}{ Category } & \multicolumn{4}{|c|}{ Upper Urinary Tract Urothelial Carcinoma } & \multicolumn{4}{|c|}{ Urinary Bladder Urothelial Carcinoma } \\
\hline & & \multirow{2}{*}{ Case No. } & \multicolumn{2}{|c|}{ ROR2 Expression } & \multirow{2}{*}{$p$-Value } & \multirow{2}{*}{ Case No. } & \multicolumn{2}{|c|}{ ROR2 Expression } & \multirow{2}{*}{$p$-Value } \\
\hline & & & Low & High & & & Low & High & \\
\hline Gender & Female & 182 & 99 & 83 & & 79 & 37 & 42 & \\
\hline \multirow{2}{*}{ Age (years) } & $<65$ & 138 & 69 & 69 & 1.000 & 121 & 59 & 62 & 0.759 \\
\hline & $\geq 65$ & 202 & 101 & 101 & & 174 & 88 & 86 & \\
\hline \multirow{3}{*}{ Tumor location } & Renal pelvis & 141 & 63 & 78 & 0.232 & - & - & - & - \\
\hline & Ureter & 150 & 82 & 68 & & - & - & - & - \\
\hline & Renal pelvis \& ureter & 49 & 25 & 24 & & - & - & - & - \\
\hline \multirow{2}{*}{ Multifocality } & Single & 278 & 137 & 141 & 0.574 & - & - & - & - \\
\hline & Multifocal & 62 & 33 & 29 & & - & - & - & - \\
\hline \multirow[b]{2}{*}{ Primary tumor $(\mathrm{T})$} & $\mathrm{Ta}$ & 89 & 57 & 32 & $<0.001 *$ & 84 & 56 & 28 & $<0.001$ * \\
\hline & $\mathrm{T} 1$ & 92 & 54 & 38 & & 88 & 43 & 45 & \\
\hline \multirow{2}{*}{ Nodal metastasis } & Negative (N0) & 312 & 165 & 147 & $<0.001 *$ & 266 & 136 & 130 & 0.177 \\
\hline & Positive (N1-N2) & 28 & 5 & 23 & & 29 & 11 & 18 & \\
\hline \multirow{2}{*}{ Histological grade } & Low grade & 56 & 37 & 19 & $0.008^{*}$ & 56 & 40 & 16 & $<0.001$ * \\
\hline & High grade & 284 & 133 & 151 & & 239 & 107 & 132 & \\
\hline \multirow{2}{*}{ Vascular invasion } & Absent & 234 & 138 & 96 & $<0.001$ * & 246 & 129 & 117 & $0.045 *$ \\
\hline & Present & 106 & 32 & 74 & & 49 & 18 & 31 & \\
\hline \multirow{2}{*}{ Perineural invasion } & Absent & 321 & 162 & 159 & 0.479 & 275 & 139 & 136 & 0.362 \\
\hline & Present & 19 & 8 & 11 & & 20 & 8 & 12 & \\
\hline \multirow{2}{*}{$\begin{array}{l}\text { Mitotic rate (per } 10 \text { high } \\
\text { power fields) }\end{array}$} & $<10$ & 173 & 92 & 81 & 0.233 & 139 & 71 & 68 & 0.686 \\
\hline & $\geq 10$ & 167 & 78 & 89 & & 156 & 76 & 80 & \\
\hline
\end{tabular}




\subsection{Prognostic Significance of ROR2 Immunoexpression}

In the UTUC cohort, the median follow-up time was 38.2 months (mean $44.7 \pm 31.9 \mathrm{~ms}$ ). During this period, 61 patients (25.2\%) experienced cancer-related death, and 70 patients $(34.5 \%)$ had tumor metastasis. Kaplan-Meier analysis revealed that patients with high ROR2 expression had a lower probability of DSS ( $p<0.0001$; Figure 3A) and MFS $(p<0.0001$; Figure 3B) compared to those with low ROR2 expression. In the multivariable analyses (Table 3), adjusted for the effects of standard clinicopathological features, demonstrated that ROR2 expression was significantly associated with the probability of disease-specific mortality (HR:3.302; 95\% CI $=1.621-6.727 ; p=0.001$ ) and metastasis (HR:6.691; 95\% CI = 3.181-14.075; $p<0.001$ ).

In the UBUC cohort, the median follow-up time was 23.4 months (mean $30.8 \pm 25.6 \mathrm{~ms}$ ). Over a median follow-up of 23.4 months, 76 patients (26.7\%) experienced cancer metastasis, and 52 (18.2\%) died subsequently from UBUC. As shown in Figure 3, high ROR2 expression was significantly associated with worse MFS $(p<0.0001$; Figure $3 C)$ and DSS $(p<0.0001$; Figure 3D) in Kaplan-Meier analysis. Predictors of oncologic outcomes identified using Cox regression analyses are summarized in Table 4 for DSS and MFS. Inthe multivariate analysis, after adjusting for primary tumor stage, tumor grade, perineural invasion, vascular invasion, lymph node status, and mitotic rate for MFS and CSS, we found that ROR2 expression status remained an independent predictor of CSS (HR:2.166; $95 \% \mathrm{CI}=1.178-3.983 ; p=0.013$ ) and MFS (HR: 2.786; 95\% CI $=1.646-4.714 ; p<0.001)$. The other independent predictors of DSS and MFS are listed in Table 4.

We performed a subgroup analysis in 172 patients with NMIBC. Of these patients, $65(37.8 \%)$ had urinary bladder tumor recurrence. In Kaplan-Meier analysis, ROR2 high expression was associated with a high urinary bladder tumor recurrence rate $(p<0.0001$; Figure 3E). In the multivariate analysis, we found that ROR2 expression status (HR: 3.033; $95 \% \mathrm{CI}=1.758-5.233, p<0.001$ ) and primary tumor stage and grade were independent predictors for urinary bladder tumor recurrence in patients with NMIBC (Table 5).

\subsection{GO Enrichment Analysis of ROR2 Co-Expressed Genes in UC}

ROR2 co-expressed genes were calculated by analyzing their mRNA expression using the cBioPortal online tool for TCGA-BLCA with Spearman's correlation. The top 500 positive and 500 negative co-expressed genes are listed in Tables S2 and S3. GO enrichment analysis was performed using the gene ontology resource online tool (Tables S4 and S5). For the biological process function, the genes were mainly enriched in fatty acid, monocarboxylic acid, and carboxylic acid metabolic processes, negative regulation of neutrophil migration, and negative regulation of granulocyte and neutrophil chemotaxis. Taken together, these results suggest that ROR2 may play crucial biological roles in UC metabolic reprogramming and immune microenvironment regulation. 
Table 3. Univariate log-rank and multivariate analyses for disease-specific and metastasis-free survival in upper urinary tract urothelial carcinoma.

\begin{tabular}{|c|c|c|c|c|c|c|c|c|c|c|c|c|}
\hline \multirow{3}{*}{ Parameter } & \multirow{3}{*}{ Category } & \multirow{3}{*}{ Case No. } & \multicolumn{5}{|c|}{ Disease-Specific Survival } & \multicolumn{5}{|c|}{ Metastasis-Free Survival } \\
\hline & & & \multicolumn{2}{|c|}{ Univariate Analysis } & \multicolumn{3}{|c|}{ Multivariate Analysis } & \multicolumn{2}{|c|}{ Univariate Analysis } & \multicolumn{3}{|c|}{ Multivariate Analysis } \\
\hline & & & No. of Event & $p$-Value & R.R. & $95 \% \mathrm{CI}$ & $p$-Value & No. of Event & $p$-Value & R.R. & $95 \% \mathrm{CI}$ & $p$-Value \\
\hline Gender & Male & 158 & 28 & 0.8286 & - & - & - & 32 & 0.7904 & - & - & - \\
\hline \multirow{2}{*}{ Age (years) } & $<65$ & 138 & 26 & 0.9943 & - & - & - & 30 & 0.8470 & - & - & - \\
\hline & $\geq 65$ & 202 & 35 & & - & - & - & 40 & & - & - & - \\
\hline \multirow{2}{*}{ Tumor side } & Right & 177 & 34 & 0.7366 & - & - & - & 38 & 0.3074 & - & - & - \\
\hline & Bilateral & 9 & 1 & & - & - & - & 0 & & - & - & - \\
\hline \multirow{3}{*}{ Tumor location } & Renal pelvis & 141 & 24 & $0.0079 *$ & 1 & - & 0.934 & 31 & 0.0659 & - & - & - \\
\hline & Ureter & 150 & 22 & & 0.864 & $0.467-1.601$ & & 25 & & - & - & - \\
\hline & Renal pelvis\& ureter & 49 & 15 & & 1.430 & $0.392-5.215$ & & 14 & & - & - & - \\
\hline \multirow{2}{*}{ Multifocality } & Single & 273 & 48 & $0.0026^{*}$ & 1 & - & $0.005^{*}$ & 52 & $0.0127^{*}$ & 1 & - & $<0.001$ * \\
\hline & Multifocal & 62 & 18 & & 3.026 & $1.400-6.539$ & & 18 & & 2.897 & $1.657-5.065$ & \\
\hline \multirow[b]{2}{*}{ Primary tumor $(\mathrm{T})$} & $\mathrm{Ta}$ & 89 & 2 & $<0.0001^{*}$ & 1 & - & $0.035 *$ & 4 & $<0.0001$ * & 1 & - & $0.037^{*}$ \\
\hline & $\mathrm{T} 1$ & 92 & 9 & & 4.810 & $1.009-22.935$ & & 15 & & 4.390 & $1.403-13.739$ & \\
\hline \multirow{2}{*}{ Nodal metastasis } & Negative (N0) & 312 & 42 & $<0.0001 *$ & 1 & - & $<0.001 *$ & 55 & $<0.0001$ * & 1 & - & 0.010 * \\
\hline & Positive (N1-N2) & 28 & 19 & & 4.062 & $2.187-7.535$ & & 15 & & 2.262 & $1.217-4.205$ & \\
\hline \multirow{2}{*}{ Histological grade } & Low grade & 56 & 4 & $0.0215^{*}$ & 1 & - & 0.052 & 3 & $0.0027^{*}$ & 1 & - & 0.265 \\
\hline & High grade & 284 & 57 & & 2.701 & $0.933-7.348$ & & 67 & & 1.592 & $0.703-3.606$ & \\
\hline \multirow{2}{*}{ Vascular invasion } & Absent & 234 & 24 & $<0.0001 *$ & 1 & - & 0.309 & 26 & $<0.0001$ * & 1 & - & $0.016^{*}$ \\
\hline & Present & 106 & 37 & & 1.371 & $0.746-2.518$ & & 44 & & 2.186 & $1.156-4.132$ & \\
\hline \multirow{2}{*}{ Perineural invasion } & Absent & 321 & 50 & $<0.0001^{*}$ & 1 & - & $<0.001 *$ & 61 & $<0.0001$ * & 1 & - & 0.001 * \\
\hline & Present & 19 & 11 & & 4.753 & $2.274-9.931$ & & 9 & & 3.634 & $1.706-7.740$ & \\
\hline \multirow{2}{*}{$\begin{array}{l}\text { Mitotic rate (per } 10 \text { high } \\
\text { power fields) }\end{array}$} & $<10$ & 173 & 27 & 0.167 & - & - & & 30 & 0.0823 & - & - & \\
\hline & $\geq 10$ & 167 & 34 & & - & - & & 40 & & - & - & \\
\hline \multirow{2}{*}{ ROR2 expression } & Low & 170 & 11 & $<0.0001^{*}$ & 1 & - & $0.001 *$ & 9 & $<0.0001 *$ & 1 & - & $<0.001$ * \\
\hline & High & 170 & 45 & & 3.302 & $1.621-6.727$ & & 61 & & 6.691 & $3.181-14.075$ & \\
\hline
\end{tabular}


Table 4. Univariate log-rank and multivariate analyses for disease-specific and metastasis-free survival in urinary bladder urothelial carcinoma.

\begin{tabular}{|c|c|c|c|c|c|c|c|c|c|c|c|c|}
\hline \multirow{3}{*}{ Parameter } & \multirow{3}{*}{ Category } & \multirow{3}{*}{ Case No. } & \multicolumn{5}{|c|}{ Disease-Specific Survival } & \multicolumn{5}{|c|}{ Metastasis-Free Survival } \\
\hline & & & \multicolumn{2}{|c|}{ Univariate Analysis } & \multicolumn{3}{|c|}{ Multivariate Analysis } & \multicolumn{2}{|c|}{ Univariate Analysis } & \multicolumn{3}{|c|}{ Multivariate Analysis } \\
\hline & & & No. of Event & $p$-Value & R.R. & $95 \% \mathrm{CI}$ & $p$-Value & No. of Event & $p$-Value & R.R. & $95 \% \mathrm{CI}$ & $p$-Value \\
\hline \multirow{2}{*}{ Gender } & Male & 216 & 41 & 0.4446 & - & - & - & 60 & 0.2720 & - & - & - \\
\hline & Female & 79 & 11 & & - & - & - & 16 & & - & - & - \\
\hline \multirow{2}{*}{ Age (years) } & $<65$ & 121 & 17 & 0.1136 & - & - & - & 31 & 0.6875 & - & - & - \\
\hline & $\geq 65$ & 174 & 35 & & - & - & - & 45 & & - & - & - \\
\hline \multirow{2}{*}{ Primary tumor $(\mathrm{T})$} & $\mathrm{Ta}$ & 84 & 1 & $<0.0001 *$ & 1 & - & $<0.001 *$ & 4 & $<0.0001^{*}$ & 1 & - & $0.001 *$ \\
\hline & $\mathrm{T} 2-\mathrm{T} 4$ & 123 & 42 & & 24.526 & $2.818-213.432$ & & 49 & & 7.448 & $2.182-25.426$ & \\
\hline \multirow{2}{*}{ Nodal metastasis } & Negative (NO) & 266 & 41 & $0.0002 *$ & 1 & - & 0.194 & 61 & $<0.0001^{*}$ & 1 & - & 0.167 \\
\hline & Positive (N1-N2) & 29 & 11 & & 1.602 & $0.787-3.263$ & & 15 & & 2.108 & $1.132-3.925$ & \\
\hline \multirow{2}{*}{ Histological grade } & Low grade & 56 & 2 & $0.0013^{*}$ & 1 & - & 0.969 & 5 & $0.0007^{*}$ & 1 & - & 0.614 \\
\hline & High grade & 239 & 50 & & 0.970 & $0.211-4.462$ & & 71 & & 1.031 & $0.365-2.910$ & \\
\hline \multirow{2}{*}{ Vascular invasion } & Absent & 246 & 37 & $0.0024^{*}$ & 1 & - & 0.136 & 54 & $0.0001 *$ & 1 & - & 0.928 \\
\hline & Present & 49 & 15 & & 0.581 & $0.285-1.187$ & & 22 & & 0.935 & $0.507-1.724$ & \\
\hline Perineural invasion & Absent & 275 & 44 & $0.0001 *$ & 1 & - & 0.067 & 66 & $0.0007 *$ & 1 & - & 0.326 \\
\hline \multirow{2}{*}{$\begin{array}{l}\text { Mitotic rate (per } 10 \text { high } \\
\quad \text { power fields) }\end{array}$} & $<10$ & 139 & 12 & $<0.0001$ * & 1 & - & $0.013 *$ & 23 & $<0.0001$ * & 1 & - & 0.012 * \\
\hline & $\geq 10$ & 156 & 40 & & 2.381 & $1.198-4.732$ & & 53 & & 1.967 & $1.162-3.329$ & \\
\hline \multirow{2}{*}{ ROR2 expression } & Low & 147 & 9 & $<0.0001 *$ & 1 & - & $0.013 *$ & 19 & $<0.0001 *$ & 1 & - & $<0.001$ * \\
\hline & High & 148 & 43 & & 2.166 & $1.178-3.983$ & & 57 & & 2.786 & $1.646-4.714$ & \\
\hline
\end{tabular}

* Statistically significant. 
Table 5. Univariate log-rank and multivariate analyses for bladder recurrence-free survival in NMIBC post TURBT.

\begin{tabular}{|c|c|c|c|c|c|c|c|}
\hline \multirow{3}{*}{ Parameter } & \multirow{3}{*}{ Category } & \multirow{3}{*}{ Case No. } & \multicolumn{5}{|c|}{ Bladder Recurrence-Free Survival } \\
\hline & & & \multicolumn{2}{|c|}{ Univariate Analysis } & \multicolumn{3}{|c|}{ Multivariate Analysis } \\
\hline & & & No. of Event & $p$-Value & R.R. & $95 \% \mathrm{CI}$ & $p$-Value \\
\hline \multirow{2}{*}{ Gender } & Male & 125 & 46 & 0.3370 & - & - & - \\
\hline & Female & 47 & 19 & & - & - & - \\
\hline \multirow{2}{*}{ Age (years) } & $<65$ & 70 & 30 & 0.3857 & - & - & - \\
\hline & $\geq 65$ & 102 & 35 & & - & - & - \\
\hline \multirow{2}{*}{ Primary tumor $(\mathrm{T})$} & $\mathrm{Ta}$ & 84 & 27 & $0.0193 *$ & 1 & - & 0.765 \\
\hline & $\mathrm{T} 1$ & 88 & 38 & & 1.098 & $0.597-2.019$ & \\
\hline \multirow{2}{*}{ Histological grade } & Low grade & 54 & 15 & $0.0101 *$ & 1 & - & 0.261 \\
\hline & High grade & 118 & 50 & & 1.513 & $0.735-3.115$ & \\
\hline \multirow{2}{*}{ Vascular invasion } & Absent & 171 & 65 & 0.6639 & - & - & - \\
\hline & Present & 1 & 0 & & - & - & - \\
\hline \multirow{2}{*}{ Perineural invasion } & Absent & 169 & 64 & 0.4725 & - & - & - \\
\hline & Present & 3 & 1 & & - & - & - \\
\hline \multirow{2}{*}{ Mitotic rate (per 10 high power fields) } & $<10$ & 94 & 35 & 0.1853 & - & - & - \\
\hline & $\geq 10$ & 78 & 30 & & - & - & - \\
\hline \multirow{2}{*}{ ROR2 expression } & Low & 99 & 20 & $<0.0001 *$ & 1 & - & $<0.001$ * \\
\hline & High & 73 & 45 & & 3.033 & $1.758-5.233$ & \\
\hline
\end{tabular}

* Statistically significant.
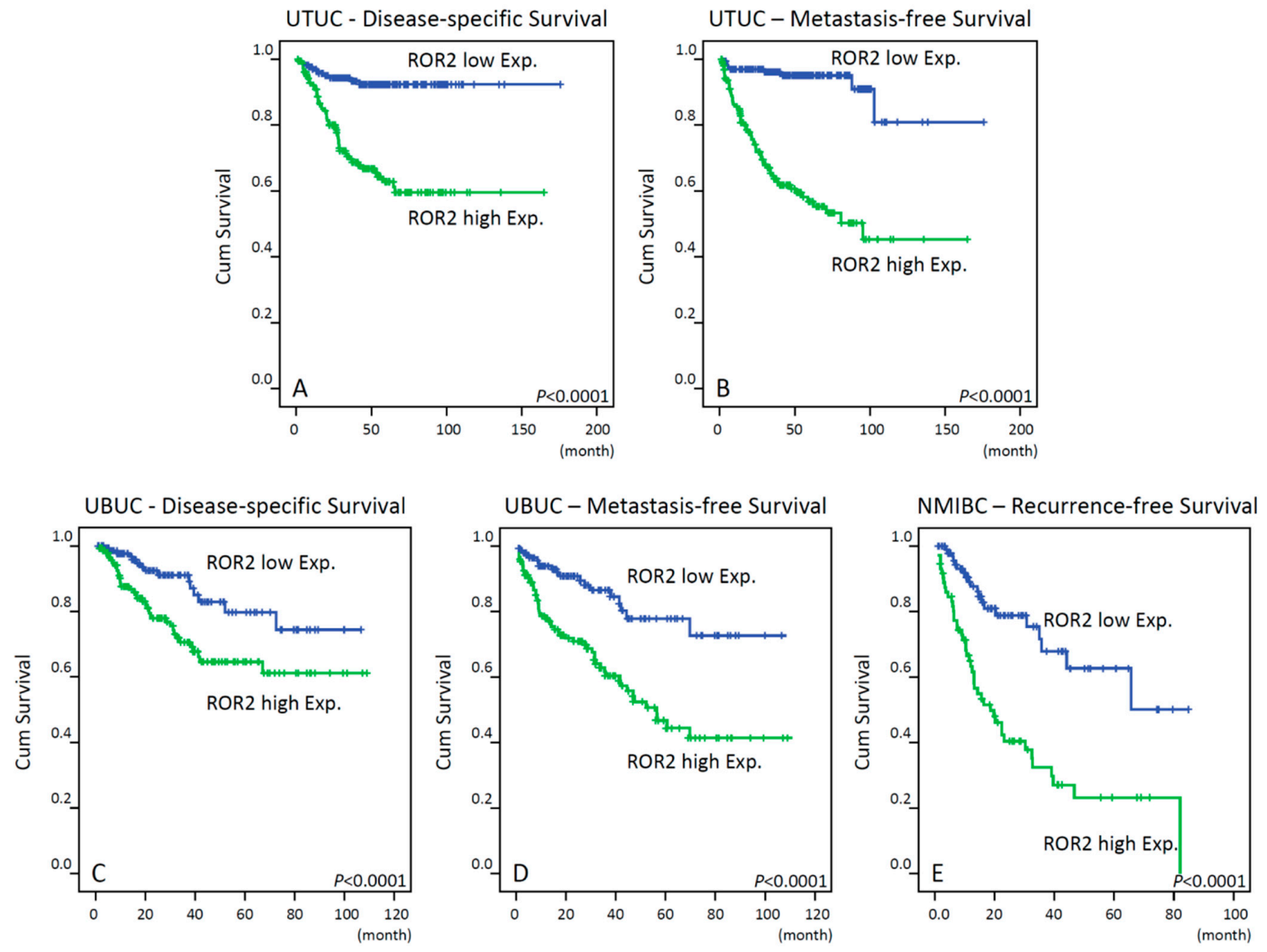

Figure 3. Kaplan-Meier survival analysis shows that ROR2 overexpression has significant prognostic impacts in diseasespecific survival, metastasis-free survival, and bladder recurrence-free survival of patients with UTUC (A,B, respectively) and UBUC (C,D, respectively) and bladder recurrence-free survival of patients with NMIBC (E). 


\section{Discussion}

$\mathrm{UC}$ is one of the most frequently diagnosed, heterogeneous, and harmful cancers worldwide. Some tissue-based biomarkers have also been assessed for their prognostic value in UC, but little is utilized routinely in clinical practice to guide individual treatment decisions. In this study, we assessed the association of ROR2 expression with the pathological features of UTUC and UBIC and its value in prognosticating DSS, MFS, and BRFS BCR in a large UC cohort. We found that ROR2 high expression was associated with established features of biologically and clinically aggressive UC, including high pathologic stage, high histological grade, nodal metastasis, vascular invasion, perineural invasion, and high mitotic activity which are often linked to poor clinical outcomes. ROR2 expression in UC may define a subset of high-risk tumors, which may require additional treatment.

The association between ROR2 expression and patient outcomes has been studied in some malignancies. Edris et al. determined whether ROR2 expression using IHC was associated with patient outcomes in sarcomas [22]. They found that ROR2 immunoexpression predicts poor overall survival and DSS in patients with gastrointestinal stromal tumor and leiomyosarcoma. However, in a multivariate analysis, ROR2 was not independent of other clinicopathological characteristics [22]. Gou et al. found that ROR2 gene mRNA expression was significantly increased in breast cancer tissues compared with corresponding non-tumor tissues [23]. High ROR2 gene mRNA expression had a large tumor size and reducedthe disease-free survival rate. Henry et al. showed ROR2 protein expression in most breast cancer patients (87\%) but not in normal breast tissue [24]. Its high expression confers a poorer DSS, especially in triple-negative breast cancer patients. In lung cancer, Lu et al. showed that ROR2 mRNA expression and protein were significantly increased in lung cancer [25]. Patients with high ROR2 expression are significantly associated with advanced cancer stage, lymph node metastasis, and a poorer survival rate after the operation. In this study, we found that ROR2 expression was associated with recurrence, metastases spread, and cancer-specific mortality in univariate analysis; moreover, after adjusting for standard prognostic factors in multivariate analysis, the ROR2 expression status remained associated with poor outcomes, implying its prognostic value.

In UBUC, for effectively treating NMIBC, the identification of high-risk patients who may progress to MIBC is a critical challenge [2,3]. We observed that ROR2 immunoexpression may be a biomarker for identifying these patients, as significantly high ROR2 expression was observed in muscle-invasive and high-grade UBUC. Patients with high ROR2 expression have three-times greater bladder tumor recurrence rate than those with low ROR2 expression, suggesting its prognostic role in NMIBC. The early identification of the patients with metastatic potential is another important issue. Our results demonstrated that UBUC patients with high ROR2 expression and aggressive pathological features were more likely to develop lymph node and distant metastasis. Patients with high ROR2expressing UBUCs may receive more aggressive management, such as early radical surgery and perioperative systemic chemotherapy or immunotherapy.

In UTUC, kidney-sparing surgery is suggested for low-risk cancers due to the low surgical complication rate and comparable survival rate to RNU [4]. Along with our findings, high ROR2-expressing UTUC is associated with aggressive cancer characteristics and worse outcomes. Therefore, RNU should be considered in low-risk UTUC patients but with high ROR2 immunoexpression. Lymphadenectomy and adjuvant/neoadjuvant chemotherapy improve local recurrence and survival rate in patients with advanced UTUC [4,26]. We found that high ROR2 expression tumors were significantly associated with muscleinvasive or lymph node metastatic UTUC and a high cancer death rate. These high-risk UTUC patients may receive an aggressive treatment protocol, RNU with lymphadenectomy, and perioperative systemic chemotherapy.

Although the detailed molecular mechanism of ROR2 in UC progression is not well understood, some regulatory pathways have been discovered in other malignancies. Many studies demonstrate that ROR2 activates WNT signaling responses by binding the WNT5A ligand $[12,27]$. Furthermore, ROR2 facilitates breast cancer progression by regulating the 
expression of PI3K/AKT and apoptotic signaling genes [4]. In detail, ROR2 overexpression promotes PI3K activation and AKT phosphorylation, followed by the downregulation of p21 and upregulation of cyclin D1 and PDK1, resulting in increased proliferation and survival of breast cancer cells [23]. ROR2 also regulates the epithelial-mesenchymal transition phenotype of breast cancer cells via the activation of the MAPK/p38 signaling pathway [28]. The overexpression of ROR2 significantly upregulated snail, N-cadherin, and vimentin but reduced E-cadherin expression [28].

The unique and critical role of the ROR2 makes it an ideal target for therapeutic intervention [27]. The ongoing study of ROR2 targeting therapy deals with adoptive immunity. Peng et al. designed a rabbit monoclonal antibody XBR2-401, which has valuable preclinical results owing to the similarity of the extracellular domains of rabbit and human ROR2. Afterward, they developed the monoclonal antibody into a chimeric antigen receptor (CAR) $\mathrm{T}$ cell format and designed the T cell-engaging ROR2 $\times$ CD3 bispecific antibodies (biAbs), which had a high specificity towards ROR2 [29,30]. Advanced investigations regarding ROR2-targeting antibody-drug conjugates are already underway. One potential candidate is BA3021, a conditionally active biological (CAB) ROR2-targeted antibody-drug conjugate (CAB-ROR2-ADC). It is now being assessed in a multicenter, open-label, phase $1 / 2$ study in patients with advanced solid tumors (NCT03504488). Two additional active clinical trials are ongoing to investigate ROR2-specific CAR T cells in ROR2-positive renal cell carcinomas (NCT03393936) and ROR2 expressing solid malignancies (NCT03960060).

JNKs are important genes in the JNK cascade. There are three differently spliced genes of JNK, namely, JNK1(MAPK8), JNK2 (MAPK9), and JNK3(MAPK10) [9]. The difference between ROR2 and JNKs is that ROR2 is a receptor protein tyrosine kinase and type I transmembrane protein, while JNKs are mitogen-activated protein kinases and intracellular proteins $[9,12]$. In our analysis, JNK3(MAPK10)there was a negative association with UC invasiveness (Table 1). Using GEPIA, we evaluated the prognostic roles of JNK1, JNK2, and JNK3 in UC. The results showed JNKs genes expression status was not associated with overall survival (Figure S2). JNK could have both oncogenic and tumor suppressing roles depending on the cancer type and stage [9,31]. Currently, most studies use pan-JNK inhibitorsin vitro, which lack specificity and indiscriminately inhibit phosphorylation of all JNK substrates [8,32]. Due to lack of efficacy and unpredicted side effects, most clinical trials of JNK inhibitors were unsuccessful [8,32]. Therefore, the development of selective JNK inhibitors are wanted to be a valuable cancer therapeutic strategy.

There are some limitations in our study, including those inherent to any retrospective study. Another limitation is that IHC results may be inconsistent due to the variability in the methodology and scoring protocols. We used a semiquantitative method, combining staining density and percentage, to evaluate immune expression activity by two independent pathologists to maximize the reliability of the results. IHC is also an inexpensive test and is available in most laboratories. Moreover, we did not elucidate the clear molecular mechanism of ROR2 and the therapeutic efficacy of ROR2 targeting therapy in UC.Further researches are needed to focus on these issues.Finally, although our UC cohort number was quite large, further validation may be necessary to confirm our results in a multi-institutional prospective design.

\section{Conclusions}

High ROR2 expression is associated with aggressive clinicopathological features in patients with UTUC and UBUC treated with curative intent. This is the first study to demonstrate that ROR2 expression is an independent prognosticator of DSS, MFS, and BRFS in UC after adjustment with standard pathological characteristics. The assessment of ROR2 expression by standard immunostaining appears to be valuable in clinical practice for patient stratification. ROR's biological role in UC pathogenesis remains to be elucidated and may be helpful as a therapeutic target. 
Supplementary Materials: The following are available online at https: / www.mdpi.com/article/ 10.3390/biomedicines9081054/s1. Table S1: The genes belonging to the JNK cascade (GO:0007254). Table S2: The top 500 most significant differentially expressed genes that are positively correlated with ROR2 expression in bladder cancer. Table S3: The top 500 most significant differentially expressed genes that are negatively correlated with ROR2 expression in bladder cancer. Table S4: GO enrichments of positively associated genes. Table S5: GO enrichments of negatively associated genes. Figure S1: Kaplan-Meier analysis of overall survival according to MAPK10, MINK1, MAP4K3, $C D C 42 E P 5$, and PAK1 expression in UC patients. Figure S2: Kaplan-Meier analysis of overall survival according to JNKs expression in UC patients.

Author Contributions: Conception and design: W.-M.L., C.-F.Y. and T.-C.C.; acquisition of data: C.-F.Y., T.-J.C., T.-C.C., L.-C.W., C.-F.L., H.-L.K., Y.-C.W., H.-Y.L., W.-J.W. and W.-M.L.; analysis and interpretation of data: W.-M.L., C.-F.L. and T.-C.C.; drafting of the manuscript: C.-F.Y. and W.-M.L.; critical revision of the manuscript: C.-F.L., W.-M.L. and H.-L.K.; statistical analysis: H.-L.K. and C.-F.L. All authors have read and agreed to the published version of the manuscript.

Funding: This research was funded by Kaohsiung Medical University Hospital, Taiwan (KMUH1077R58, KMUH108-8M53, KMUH-DK(C)-110006) and Ministry of Science and Technology, Taiwan (MOST109-2314-B-037-110-MY3, MOST110-2321-B-037-002).

Institutional Review Board Statement: This study was approved by the Institutional Review Board of the Chi Mei Medical Center (IRB10501-005) on 4 February 2016.

Informed Consent Statement: Informed consent was obtained from all subjects involved in the study.

Data Availability Statement: All data generated or analyzed during this study are included in this published article and its Supplementary Documentation File.

Conflicts of Interest: The authors declare no conflict of interest. The funders had no role in the design of the study; in the collection, analyses, or interpretation of data; in the writing of the manuscript, or in the decision to publish the results.

\section{References}

1. Bray, F.; Ferlay, J.; Soerjomataram, I.; Siegel, R.L.; Torre, L.A.; Jemal, A. Global cancer statistics 2018: GLOBOCAN estimates of incidence and mortality worldwide for 36 cancers in 185 countries. CA Cancer J. Clin. 2018, 68, 394-424. [CrossRef]

2. Babjuk, M.; Burger, M.; Comperat, E.M.; Gontero, P.; Mostafid, A.H.; Palou, J.; van Rhijn, B.W.G.; Roupret, M.; Shariat, S.F.; Sylvester, R.; et al. European Association of Urology Guidelines on Non-muscle-invasive Bladder Cancer (TaT1 and Carcinoma In Situ)-2019 Update. Eur. Urol. 2019, 76, 639-657. [CrossRef] [PubMed]

3. Witjes, J.A.; Bruins, H.M.; Cathomas, R.; Comperat, E.M.; Cowan, N.C.; Gakis, G.; Hernandez, V.; Linares Espinos, E.; Lorch, A.; Neuzillet, Y.; et al. European Association of Urology Guidelines on Muscle-invasive and Metastatic Bladder Cancer: Summary of the 2020 Guidelines. Eur. Urol. 2021, 79, 82-104. [CrossRef] [PubMed]

4. Roupret, M.; Babjuk, M.; Burger, M.; Capoun, O.; Cohen, D.; Comperat, E.M.; Cowan, N.C.; Dominguez-Escrig, J.L.; Gontero, P.; Hugh Mostafid, A.; et al. European Association of Urology Guidelines on Upper Urinary Tract Urothelial Carcinoma: 2020 Update. Eur. Urol. 2021, 79, 62-79. [CrossRef]

5. van Osch, F.H.; Jochems, S.H.; van Schooten, F.J.; Bryan, R.T.; Zeegers, M.P. Quantified relations between exposure to tobacco smoking and bladder cancer risk: A meta-analysis of 89 observational studies. Int. J. Epidemiol. 2016, 45, 857-870. [CrossRef] [PubMed]

6. Figueroa, J.D.; Middlebrooks, C.D.; Banday, A.R.; Ye, Y.; Garcia-Closas, M.; Chatterjee, N.; Koutros, S.; Kiemeney, L.A.; Rafnar, T.; Bishop, T.; et al. Identification of a novel susceptibility locus at 13q34 and refinement of the 20p12.2 region as a multi-signal locus associated with bladder cancer risk in individuals of European ancestry. Hum. Mol. Genet. 2016, 25, 1203-1214. [CrossRef]

7. Martin, C.; Leiser, C.L.; O'Neil, B.; Gupta, S.; Lowrance, W.T.; Kohlmann, W.; Greenberg, S.; Pathak, P.; Smith, K.R.; Hanson, H.A. Familial Cancer Clustering in Urothelial Cancer: A Population-Based Case-Control Study. J. Natl. Cancer Inst. 2018, 110, 527-533. [CrossRef] [PubMed]

8. Bubici, C.; Papa, S. JNK signalling in cancer: In need of new, smarter therapeutic targets. Br. J. Pharmacol 2014, 171, 24-37. [CrossRef]

9. Semba, T.; Sammons, R.; Wang, X.; Xie, X.; Dalby, K.N.; Ueno, N.T. JNK Signaling in Stem Cell Self-Renewal and Differentiation. Int. J. Mol. Sci. 2020, 21, 2613. [CrossRef]

10. Zeke, A.; Misheva, M.; Remenyi, A.; Bogoyevitch, M.A. JNK Signaling: Regulation and Functions Based on Complex ProteinProtein Partnerships. Microbiol. Mol. Biol. Rev. 2016, 80, 793-835. [CrossRef]

11. Papa, S.; Choy, P.M.; Bubici, C. The ERK and JNK pathways in the regulation of metabolic reprogramming. Oncogene 2019, 38, 2223-2240. [CrossRef] [PubMed] 
12. Debebe, Z.; Rathmell, W.K. Ror2 as a therapeutic target in cancer. Pharmacol. Ther. 2015, 150, 143-148. [CrossRef]

13. Morioka, K.; Tanikawa, C.; Ochi, K.; Daigo, Y.; Katagiri, T.; Kawano, H.; Kawaguchi, H.; Myoui, A.; Yoshikawa, H.; Naka, N.; et al. Orphan receptor tyrosine kinase ROR2 as a potential therapeutic target for osteosarcoma. Cancer Sci. 2009, 100, 1227-1233. [CrossRef]

14. Yamamoto, H.; Oue, N.; Sato, A.; Hasegawa, Y.; Yamamoto, H.; Matsubara, A.; Yasui, W.; Kikuchi, A. Wnt5a signaling is involved in the aggressiveness of prostate cancer and expression of metalloproteinase. Oncogene 2010, 29, 2036-2046. [CrossRef] [PubMed]

15. Wright, T.M.; Brannon, A.R.; Gordan, J.D.; Mikels, A.J.; Mitchell, C.; Chen, S.; Espinosa, I.; van de Rijn, M.; Pruthi, R.; Wallen, E.; et al. Ror2, a developmentally regulated kinase, promotes tumor growth potential in renal cell carcinoma. Oncogene 2009, 28, 2513-2523. [CrossRef] [PubMed]

16. Lara, E.; Calvanese, V.; Huidobro, C.; Fernandez, A.F.; Moncada-Pazos, A.; Obaya, A.J.; Aguilera, O.; Gonzalez-Sancho, J.M.; Sanchez, L.; Astudillo, A.; et al. Epigenetic repression of ROR2 has a Wnt-mediated, pro-tumourigenic role in colon cancer. Mol. Cancer 2010, 9, 170. [CrossRef] [PubMed]

17. Geng, M.; Cao, Y.C.; Chen, Y.J.; Jiang, H.; Bi, L.Q.; Liu, X.H. Loss of Wnt5a and Ror2 protein in hepatocellular carcinoma associated with poor prognosis. World J. Gastroenterol. 2012, 18, 1328-1338. [CrossRef] [PubMed]

18. Fan, E.W.; Li, C.C.; Wu, W.J.; Huang, C.N.; Li, W.M.; Ke, H.L.; Yeh, H.C.; Wu, T.F.; Liang, P.I.; Ma, L.J.; et al. FGF7 Over Expression is an Independent Prognosticator in Patients with Urothelial Carcinoma of the Upper Urinary Tract and Bladder. J. Urol. 2015, 194, 223-229. [CrossRef]

19. Li, W.M.; Huang, C.N.; Ke, H.L.; Li, C.C.; Wei, Y.C.; Yeh, H.C.; Chang, L.L.; Huang, C.H.; Liang, P.I.; Yeh, B.W.; et al. MCM10 overexpression implicates adverse prognosis in urothelial carcinoma. Oncotarget 2016, 7, 77777-77792. [CrossRef]

20. Liang, P.I.; Yeh, B.W.; Li, W.M.; Chan, T.C.; Chang, I.W.; Huang, C.N.; Li, C.C.; Ke, H.L.; Yeh, H.C.; Wu, W.J.; et al. DPP4/CD26 overexpression in urothelial carcinoma confers an independent prognostic impact and correlates with intrinsic biological aggressiveness. Oncotarget 2017, 8, 2995-3008. [CrossRef] [PubMed]

21. Chang, I.W.; Lin, V.C.; Hung, C.H.; Wang, H.P.; Lin, Y.Y.; Wu, W.J.; Huang, C.N.; Li, C.C.; Li, W.M.; Wu, J.Y.; et al. GPX2 underexpression indicates poor prognosis in patients with urothelial carcinomas of the upper urinary tract and urinary bladder. World J. Urol. 2015, 33, 1777-1789. [CrossRef] [PubMed]

22. Edris, B.; Espinosa, I.; Muhlenberg, T.; Mikels, A.; Lee, C.H.; Steigen, S.E.; Zhu, S.; Montgomery, K.D.; Lazar, A.J.; Lev, D.; et al. ROR2 is a novel prognostic biomarker and a potential therapeutic target in leiomyosarcoma and gastrointestinal stromal tumour. J. Pathol. 2012, 227, 223-233. [CrossRef]

23. Guo, M.; Ma, G.; Zhang, X.; Tang, W.; Shi, J.; Wang, Q.; Cheng, Y.; Zhang, B.; Xu, J. ROR2 knockdown suppresses breast cancer growth through PI3K/ATK signaling. Aging 2020, 12, 13115-13127. [CrossRef]

24. Frenquelli, M.; Caridi, N.; Antonini, E.; Storti, F.; Vigano, V.; Gaviraghi, M.; Occhionorelli, M.; Bianchessi, S.; Bongiovanni, L.; Spinelli, A.; et al. The WNT receptor ROR2 drives the interaction of multiple myeloma cells with the microenvironment through AKT activation. Leukemia 2020, 34, 257-270. [CrossRef]

25. Lu, C.; Wang, X.; Zhu, H.; Feng, J.; Ni, S.; Huang, J. Over-expression of ROR2 and Wnt5a cooperatively correlates with unfavorable prognosis in patients with non-small cell lung cancer. Oncotarget 2015, 6, 24912-24921. [CrossRef]

26. Leow, J.J.; Chong, Y.L.; Chang, S.L.; Valderrama, B.P.; Powles, T.; Bellmunt, J. Neoadjuvant and Adjuvant Chemotherapy for Upper Tract Urothelial Carcinoma: A 2020 Systematic Review and Meta-analysis, and Future Perspectives on Systemic Therapy. Eur. Urol. 2021, 79, 635-654. [CrossRef] [PubMed]

27. Menck, K.; Heinrichs, S.; Baden, C.; Bleckmann, A. The WNT/ROR Pathway in Cancer: From Signaling to Therapeutic Intervention. Cells 2021, 10, 142. [CrossRef] [PubMed]

28. Xu, J.; Shi, J.; Tang, W.; Jiang, P.; Guo, M.; Zhang, B.; Ma, G. ROR2 promotes the epithelial-mesenchymal transition by regulating $\mathrm{MAPK} / \mathrm{p} 38$ signaling pathway in breast cancer. J. Cell Biochem. 2020, 121, 4142-4153. [CrossRef]

29. Peng, H.; Nerreter, T.; Chang, J.; Qi, J.; Li, X.; Karunadharma, P.; Martinez, G.J.; Fallahi, M.; Soden, J.; Freeth, J.; et al. Mining Naive Rabbit Antibody Repertoires by Phage Display for Monoclonal Antibodies of Therapeutic Utility. J. Mol. Biol. 2017, 429, 2954-2973. [CrossRef]

30. Goydel, R.S.; Weber, J.; Peng, H.; Qi, J.; Soden, J.; Freeth, J.; Park, H.; Rader, C. Affinity maturation, humanization, and cocrystallization of a rabbit anti-human ROR2 monoclonal antibody for therapeutic applications. J. Biol. Chem. 2020, 295, 5995-6006. [CrossRef] [PubMed]

31. Tam, S.Y.; Law, H.K. JNK in Tumor Microenvironment: Present Findings and Challenges in Clinical Translation. Cancers 2021, 13, 2196. [CrossRef] [PubMed]

32. Wu, Q.; Wu, W.; Jacevic, V.; Franca, T.C.C.; Wang, X.; Kuca, K. Selective inhibitors for JNK signalling: A potential targeted therapy in cancer. J. Enzyme Inhib. Med. Chem. 2020, 35, 574-583. [CrossRef] [PubMed] 